\title{
It's not all about muscle: fibroadipogenic progenitors contribute to facioscapulohumeral muscular dystrophy
}

\author{
Carlo Serra1,2 and Kathryn R. Wagner ${ }^{1,2,3}$ \\ 'Center for Genetic Muscle Disorders, Kennedy Krieger Institute, Baltimore, Maryland, USA. ${ }^{2}$ Department of Neurology and ${ }^{3}$ Department of Neuroscience, Johns Hopkins School of Medicine, Baltimore \\ Maryland, USA.
}

\author{
Facioscapulohumeral muscular dystrophy (FSHD) results from expression of \\ the full-length double homeobox 4 (DUX4-FL) retrogene in skeletal muscle. \\ However, even in cases of severe FSHD the presence of DUX4 is barely \\ detectable. In this issue of the $J C I$, Bosnakovski et al. used an inducible, \\ muscle-specific human DUX4 to reproduce the low-level, sporadic DUX4 \\ expression of human FSHD muscle as well the myopathology seen in \\ human FSHD disease. Notably, dysregulated fibroadipogenic progenitors \\ accumulated in affected muscles, thus providing a mechanism for the \\ replacement of muscle by fibrosis and fat.
}

\section{Facioscapulohumeral muscular dystrophy}

Facioscapulohumeral muscular dystrophy (FSHD) is a common and disabling myopathy with an incidence of approximately 1 in 8000 individuals (1). It is an autosomal dominant, genetic disease characterized by slowly progressive weakness and wasting of the muscles of the face, scapular region, upper arms, trunk, and legs. The disorder exhibits a variable onset and severity of clinical symptoms, which range from asymptomatic carriers to early-onset severe phenotypes. FSHD muscles are marked by variability in fiber size, necrosis, regeneration, decreased capillary density, and occasional lymphocytic infiltrates (2). The final common endpoint of FSHD is the replacement of muscle by fat and fibrosis, a feature shared with other muscular dystrophies (3-6).

FSHD is due to the ectopic expression of the double homeobox 4 (DUX4) retrogene in skeletal muscle. DUX4 maps onto chromosome 4 at $4 \mathrm{q} 35$ in the D4Z4 macrosatellite repeat array, which normally contains 11-100 repeats. Each D4Z4 repeat contains an open reading frame for the DUX4 gene that, when transcribed, generates two major transcripts, DUX4-s, a short nonpathogenic mRNA and a fulllength mRNA, DUX4-FL, with a translated protein that is considered the causative factor of FSHD (7-9). DUX4, as well as Dux, its murine ortholog, is a transcription factor active in the embryo at the cleavage stage to support zygotic genome activation and in primordial germ cells that is absent in the adult tissues (except in the germ cells of the testis) due to the epigenetic silencing of the D4Z4 array $(9,10)$.

There are two forms of FSHD: FSHD1 (95\% of the cases) and FSHD2 (5\% of the cases). FSHD1 is due to the contraction of the D4Z4 array, from 1 to 10 repeats, whereas patients with FSHD2, with a regular D4Z4 array, have mutations in one of two genes: structural maintenance of chromosomes flexible hinge domaincontaining protein 1 (SMCHD1) or DNA methyltransferase 3B (DNMT3B). These mutations lead to the epigenetic derepression of the D4Z4 array and thus to the tran-

Delated Article: p. 2465

Conflict of interest: KRW discloses that she is a consultant for Asklepios Biopharmaceuticals, Vita Therapeutics and Casma Therapeutics and has received research support from Sarepta Therapeutics Inc., F. Hoffmann-La Roche, Catabasis Pharmaceuticals, Fulcrum Therapeutics, PTC Therapeutics, and Pfizer Inc.

Copyright: @ 2020, American Society for Clinical Investigation.

Reference information: / Clin Invest. 2020;130(5):2186-2188. https://doi.org/10.1172/JCI136133.

scription of DUX4. The net result is the production of DUX4 by the muscle, probably due to the binding of muscle-restricted transcription factors to enhancers and promoters located upstream of the D4Z4 array $(7,8)$. Even in severe FSHD cases, the presence of DUX4 is barely detectable, indicating that DUX4-FL is sporadically transcribed and that poorly defined epigenetic mechanisms and/or genetic modifiers may control its expression. The forced expression of DUX4 in both human and mouse myoblasts in vitro leads to cell apoptosis, oxidative stress, reduced differentiation, and to the transcription of germ cell-specific genes, immune mediators genes, and retrotransposons (11-16).

\section{A long-term chronic disease model}

In this issue of the JCI, Bosnakovski et al. characterize in detail the muscle phenotype of a doxycycline-inducible human DUX4 muscle fiber-specific mouse model of FSHD, the iDUX4pA-HSA mice (17). Thanks to the adjustable level of doxycycline administered to mice, the authors reproduce the very-low-level, sporadic, DUX4 expression of the human FSHD muscle, a key point to studying the progression of FSHD myopathy in experimental models in vivo. iDUX4pA-HSA mice fed doxycycline-containing chow over 6 months showed a slowly progressive muscle atrophy, coupled with reduced muscle force-generating capacity. Histologically, the muscles of these mice showed necrosis and regeneration, infiltration of immune cells, accumulation of extracellular matrix, occasional fat deposition, decreased capillarity density, and reduced numbers of endothelial cells and pericytes, all in the presence of rare DUX4 $4^{+}$myofibers (17). Thus, the muscle phenotype of the iDUX4pA-HSA mice mimics that of the human FSHD muscle.

Bosnakovski and colleagues then compared the transcriptional profile of the 

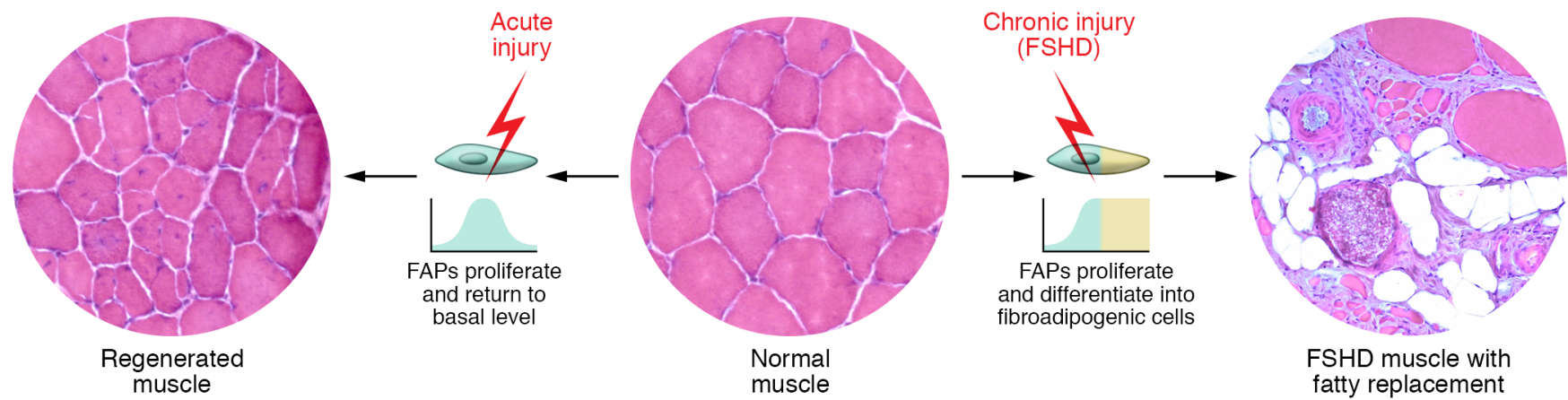

Figure 1. FAPs' potential contribution to FSHD myopathy. Upon acute muscle injury, normal muscle (center) shows necrosis of the damaged fibers and then the formation of new fibers by the fusion of the resident MuSCs (left). In this context, FAPs proliferate to assist this process and subsequently return to the basal level. Conversely, the continuous cycles of muscle necrosis and regeneration, as in FSHD, result in the excessive deposition of FAPs, a change that helps to explain the massive accumulation of fibrosis and intramuscular fat in the dystrophic muscle (right).

muscle of iDUX4pA-HSA mice treated acutely ( 2 weeks) or chronically (16 weeks) with doxycycline and identified the expression of DUX4 target genes as well as of cell death-related pathways in both treatment groups (17). Chronic DUX4 expression resulted in higher levels of dysregulation of pathways associated with the immune response. Importantly, the researchers found that the set of the differentially expressed genes (DEGs) in the chronically treated mice overlaps with that of their human homolog genes in FSHD muscle obtained from MRI-guided biopsies of short T1 inversion recovery (STIR) positivity, thought to indicate active disease $(17,18,19)$.

\section{Accumulating fibroadipogenic progenitors}

Fibroadipogenic progenitors (FAPs) are muscle interstitial mesenchymal cells that play a key role in muscle regeneration (20, 21). While the inflammatory cells recruited by the damaged muscle regulate FAPs proliferation and apoptosis, FAPs coordinate the action of the muscle satellite cells (MuSCs) to produce new myofibers (20-22), as shown by the impaired muscle regeneration after injury in FAP-depleted mice or by blocking FAP expansion by pharmacological means (22-24). This suggests that the presence of a carefully equilibrated signaling crosstalk among MuSCs, muscle fibers, and FAPs leads to successful muscle healing after injury $(20,21)$. However, in dystrophic muscle, FAPs largely terminally differentiate into fibroblasts and adipocytes $(5,22)$, an outcome that severely and irreversibly compromises the muscle physiology, leading to the common end-stage pathology of muscle, and indicating the loss of this intercellular equilibrium (refs. 3, 25 and Figure 1).

Bosnakovski et al. demonstrate that iDUX4pA-HSA mice treated with doxycycline for 3 months had a dose-dependent accumulation of intramuscular FAPs. This change correlated with the degree of muscle atrophy, the extension of the intramuscular fibrosis and inflammatory infiltrate, and the reduction in MuSCs number (17). However, it is not clear whether the reduced number of MuSCs is due to their exhaustion from chronic muscle degeneration and regeneration or whether muscle fibers, which express DUX4, can induce apoptosis in MuSCs.

The authors then performed a gene expression analysis on FAPs of the iDUX4pA-HSA mice treated with doxycycline acutely for 10 days or chronically for 6 months and found that the FAPs from the two time points share only the $22 \%$ of DEGs (17). Of note, FAPs from chronic treatment showed the downregulation of antiinflammatory signaling, suggesting that DUX4-expressing myofibers can drive a process of selection/differentiation of FAPs, making FAPs more sensitive to inflammatory stimuli during the progression of the disease (17). Here, it is important to note that the accumulation of FAPs in the muscle of chronically treated iDUX4pA-HSA mice is associated with an increased number of inflammatory cells, which release cytokines that can affect FAPs transcriptome. Interestingly, most of the DEGs of FAPs sorted from the chronically treated mice are not shared with FAPs isolated after acute muscle injury with cardiotoxin or after muscle denerva- tion (4). In this regard, while the shared DEGs, belonging to pathways controlling cell adhesion and motility, may represent a common signature of FAPs of muscles experiencing different types/degrees of injury and adaptation, the unique DEGs may represent the signature of FSHD FAPS. These DEGs may reflect the result of the inputs delivered to FAPs during the progression of the myopathy, e.g., by the unbalanced equilibrium of proinflammatory and antiinflammatory cytokines that may affect FAPs' differentiation. Of note, FAPs from both acutely and chronic treated iDUX4pA-HSA mice and FSHD muscle biopsies show comparable changes of expression between the mouse genes and their human orthologs, indicating a similarity in the progression of the FSHD myopathy between the patients and this experimental mouse model (17).

This intriguing paper highlights evidence that FAPs accumulate in DUX4expressing dystrophic muscle and do not respond to stimuli that return their numbers to a basal level, as occurs after an acute muscle injury. On the contrary, FAPs from DUX4-expressing dystrophic muscle seem to be locked in a condition that may facilitate their differentiation into fibroblasts and adipocytes. In this respect, the simultaneous presence of both proinflammatory (M1) $\mathrm{CD}^{+} 8^{+}$and antiinflammatory (M2) CD206 ${ }^{+}$ macrophages in the muscle of the chronically treated iDUX4pA-HSA mice suggests that the necrosis of the FSHD muscle could establish a dysregulated cytokine environment that allows FAPs accumulation and fibrogenic/adipogenic differentiation. Based on this evidence, it will be important to investigate whether intramuscular fibro- 
blasts and adipocytes result from the differentiation of FAPs belonging to the whole FAPs population or, instead, from the stepwise selection/differentiation of specific FAPs subpopulations, whose existence has been suggested in DMD experimental models (5). Understanding the mechanism of the massive FAPs adipogenic differentiation in the FSHD muscle is a key point for the design of new and effective therapies for FSHD, in which the common final pathology is fatty replacement.

Address correspondence to: Kathryn A. Wagner, Center for Genetic Muscle Disorders, Kennedy Krieger Institute, $707 \mathrm{~N}$. Broadway, Baltimore, Maryland 21205, USA. Phone: 443.923.9525; Email: wagnerk@ kennedykrieger.org.

1. Wagner KR. Facioscapulohumeral muscular dystrophies. Continuum (Minneap Minn). 2019;25(6):1662-1681.

2. Statland JM, et al. Immunohistochemical characterization of facioscapulohumeral muscular dystrophy muscle biopsies. J Neuromuscul Dis. 2015;2(3):291-299.

3. Janssen BH, et al. Distinct disease phases in muscles of facioscapulohumeral dystrophy patients identified by MR detected fat infiltration. PLoS One. 2014;9(1):e85416.

4. Madaro L, et al. Denervation-activated STAT3IL-6 signalling in fibro-adipogenic progenitors promotes myofibres atrophy and fibrosis. Nat Cell Biol. 2018;20(8):917-927.

5. Malecova B, et al. Dynamics of cellular states of fibro-adipogenic progenitors during myogenesis and muscular dystrophy. Nat Commun.
2018;9(1):3670.

6. Leung DG, Carrino JA, Wagner KR, Jacobs MA. Whole-body magnetic resonance imaging evaluation of facioscapulohumeral muscular dystrophy. Muscle Nerve. 2015;52(4):512-520.

7. Hewitt JE, et al. Analysis of the tandem repeat locus D4Z4 associated with facioscapulohumeral muscular dystrophy. Hum Mol Genet. 1994;3(8):1287-1295.

8. Gabriëls J, et al. Nucleotide sequence of the partially deleted D4Z4 locus in a patient with FSHD identifies a putative gene within each 3.3 kb element. Gene. 1999;236(1):25-32.

9. Snider L, et al. Facioscapulohumeral dystrophy: incomplete suppression of a retrotransposed gene. PLoS Genet. 2010;6(10):e1001181.

10. Hendrickson PG, et al. Conserved roles of mouse DUX and human DUX4 in activating cleavage-stage genes and MERVL/HERVL retrotransposons. Nat Genet. 2017;49(6):925-934.

11. Geng LN, et al. DUX4 activates germline genes, retroelements, and immune mediators: implications for facioscapulohumeral dystrophy. Dev Cell. 2012;22(1):38-51.

12. Snider L, et al. RNA transcripts, miRNA-sized fragments and proteins produced from D4Z4 units: new candidates for the pathophysiology of facioscapulohumeral dystrophy. Hum Mol Genet. 2009;18(13):2414-2430.

13. Shadle SC, et al. DUX4-induced dsRNA and MYC mRNA stabilization activate apoptotic pathways in human cell models of facioscapulohumeral dystrophy. PLoS Genet. 2017;13(3):e1006658.

14. Vanderplanck C, et al. The FSHD atrophic myotube phenotype is caused by DUX4 expression. PLOS ONE. 2011;6(10):e26820.

15. Young JM, et al. DUX4 binding to retroelements creates promoters that are active in FSHD muscle and testis. PLoS Genet. 2013;9(11):e1003947.

16. Bosnakovski D, Gearhart MD, Toso EA, Ener
ET, Choi SH, Kyba M. Low level DUX4 expression disrupts myogenesis through deregulation of myogenic gene expression. Sci Rep. 2018;8(1):16957.

17. Bosnakovski D, et al. Transcriptional and cytopathological hallmarks of FSHD in chronic DUX4-expressing mice. JClin Invest. 2020;130(5):2465-2477.

18. Tasca G, et al. Different molecular signatures in magnetic resonance imaging-staged facioscapulohumeral muscular dystrophy muscles. PLoS One. 2012;7(6):e38779.

19. Wang LH, et al. MRI-informed muscle biopsies correlate MRI with pathology and DUX4 target gene expression in FSHD. Hum Mol Genet. 2019;28(3):476-486.

20. Joe AW, et al. Muscle injury activates resident fibro/adipogenic progenitors that facilitate myogenesis. Nat Cell Biol. 2010;12(2):153-163.

21. Uezumi A, Fukada S, Yamamoto N, Takeda S, Tsuchida K. Mesenchymal progenitors distinct from satellite cells contribute to ectopic fat cell formation in skeletal muscle. Nat Cell Biol. 2010;12(2):143-152.

22. Lemos DR, et al. Nilotinib reduces muscle fibrosis in chronic muscle injury by promoting TNF-mediated apoptosis of fibro/adipogenic progenitors. Nat Med. 2015;21(7):786-794.

23. Fiore D, et al. Pharmacological blockage of fibro/adipogenic progenitor expansion and suppression of regenerative fibrogenesis is associated with impaired skeletal muscle regeneration. Stem Cell Res. 2016;17(1):161-169.

24. Wosczyna MN, et al. Mesenchymal stromal cells are required for regeneration and homeostatic maintenance of skeletal muscle. Cell Rep. 2019;27(7):2029-2035.e5.

25. Hogarth MW, et al. Fibroadipogenic progenitors are responsible for muscle loss in limb girdle muscular dystrophy 2B. Nat Commun. 2019;10(1):2430 